\title{
Wigner Intracule for the Kellner Helium-Like Ions
}

\author{
PETER M. W. GILL, NICHOLAS A. BESLEY, DARRAGH P. O'NEILL \\ School of Chemistry, University of Nottingham, Nottingham, NG7 2RD, England \\ Received 27 August 2003; accepted 20 February 2004 \\ Published online 7 June 2004 in Wiley InterScience (www.interscience.wiley.com). \\ DOI 10.1002/qua.20125
}

\begin{abstract}
The Kellner wavefunction for a helium-like ion is the Hartree-Fock solution wherein the orbital is a Slater-type function with the variationally optimal exponent $\zeta=Z-5 / 16$. The Wigner intracule $W(u, v)$ of a system gives the joint quasiprobability of finding two electrons whose position space and momentum space separations are $\left|\mathbf{r}_{1}-\mathbf{r}_{2}\right|=u$ and $\left|\mathbf{p}_{1}-\mathbf{p}_{2}\right|=v$, respectively. In this article, we extend Wigner intracule theory beyond Gaussian-type functions by deriving $W(u, v)$ for the Kellner helium-like ions. Although we have not been able to express $W(u, v)$ in closed form, our formulation reduces it to a two-dimensional integral that can be treated by quadrature. (c) 2004 Wiley Periodicals, Inc. Int J Quantum Chem 100: 166-171, 2004
\end{abstract}

\section{Introduction}

H erbert Jones devoted much of his research career to the development of methods for the incorporation of Slater-type functions (STFs), rather than Gaussian-type functions (GTFs), into molecular orbital theory (see [1-4] and references therein). It has long been appreciated that, since the exact electronic wavefunctions of molecules are known to possess cusps at the nuclei [5] and to decay exponentially at large distances [6], STFs are more appropriate basis functions than GTFs for the expansion of such wavefunctions and one probably needs fewer STFs than GTFs to generate high-quality molecular orbitals. There can therefore be little doubt that the development of highly efficient software

Correspondence to: P. Gill; e-mail: peter.gill@nottingham.ac.uk for quantum chemical calculations using STFs would be beneficial.

The Schrödinger equation (in atomic units) for a helium-like ion with nuclear charge $Z$ is

$$
\left(-\frac{\nabla_{1}^{2}}{2}-\frac{\nabla_{2}^{2}}{2}-\frac{Z}{r_{1}}-\frac{Z}{r_{2}}+\frac{1}{r_{12}}\right) \Psi=E \Psi
$$

and finding a variationally optimal wavefunction for the ground state of this system is a textbook example of the use of the variation theorem. In 1927, Kellner showed that the lowest-energy wavefunction of the "screened hydrogenic" form [7]

$$
\Psi^{\text {Kellner }}\left(\mathbf{r}_{1}, \mathbf{r}_{2}\right)=\left(\zeta^{3} / \pi\right) \exp \left(-\zeta r_{1}\right) \exp \left(-\zeta r_{2}\right)
$$

has $\zeta=Z-5 / 16$. Eckart [8], Hylleraas [9], Kinoshita [10], Pekeris [11], and many others more recently [12] have subsequently explored more so- 
phisticated approximate wavefunctions but all used this as their starting point.

We have recently initiated an investigation into the generation, properties and usefulness of a class of two-electron density functions called intracules [13-20]. The position intracule $P(u)$ is the spherically averaged probability density for the position space distance $r_{12}=\left|\mathbf{r}_{1}-\mathbf{r}_{2}\right|$ between two electrons in a quantum mechanical system, and we have shown $[13,14]$ that it can be computed efficiently for moderately large molecules. The momentum intracule $M(v)$ is the analogous probability density for the momentum space distance $p_{12}=\left|\mathbf{p}_{1}-\mathbf{p}_{2}\right|$ between two electrons, and we have shown [15] that the cost of computing $M(v)$ is roughly twice that of computing $P(u)$. The Wigner intracule $W(u$, v) can be interpreted [16] as the joint probability density for $r_{12}$ and $p_{12}$ and we have shown [17] that it is significantly more expensive to calculate than $M(v)$. The action intracule $A(w)$ can be interpreted [16] as the probability density for the product $r_{12} p_{12}$ and appears to be even more computationally demanding than $W(u, v)$. We note that both $W(u, v)$ and $A(w)$ are quasi-probability, not true probability, densities [16].

We have applied our intracule methods to study electron correlation effects in hookium and helium [18] and the effects of electronic excitation in methanal and butadiene [19]. We have also shown how the construction of the Wigner intracule can be embedded within an SCF procedure, yielding an approach that we have termed Hartree-FockWigner theory [20]. However, all of our work on Wigner intracules has pertained to wavefunctions that are expanded in GTFs, and it is interesting to examine the issues that arise when one attempts to calculate $W(u, v)$ for a wavefunction based on STFs. In this article, we report our first attempt in this direction: the calculation of the Wigner intracule for the Kellner wavefunction.

\section{Theory}

The Hartree-Fock wavefunction for a heliumlike ion is

$$
\Psi^{\mathrm{HF}}\left(\mathbf{r}_{1}, \mathbf{r}_{2}\right)=\psi\left(\mathbf{r}_{1}\right) \psi\left(\mathbf{r}_{2}\right),
$$

where $\psi(\mathbf{r})$ is a spherically symmetric spatial orbital. The resulting position, momentum, Wigner and action intracules [16] are defined by

$$
\begin{gathered}
P(u)=\int \psi(\mathbf{r})^{2} \psi(\mathbf{r}+\mathbf{u})^{2} d \mathbf{r} d \Omega_{\mathbf{u}} \\
M(v)=\int \hat{\psi}(\mathbf{p})^{2} \hat{\psi}(\mathbf{p}+\mathbf{v})^{2} d \mathbf{p} d \Omega_{\mathbf{v}} \\
W(u, v)=\frac{v^{2}}{2 \pi^{2}} \int \psi(\mathbf{r}) \psi(\mathbf{r}+\mathbf{q}) \psi(\mathbf{r}+\mathbf{q}+\mathbf{u}) \\
\times \psi(\mathbf{r}+\mathbf{u}) j_{0}(q v) d \mathbf{r} d \mathbf{q} d \Omega_{\mathbf{u}} \\
A(w)=\int_{0}^{\infty} W\left(u, \frac{w}{u}\right) \frac{d u}{u}
\end{gathered}
$$

where $\hat{\psi}(\mathbf{p})$ is the Fourier transform of $\psi(\mathbf{r})$.

If the orbital is a Gaussian function

$$
\begin{gathered}
\psi(\mathbf{r})=\left(\frac{2 \alpha}{\pi}\right)^{3 / 4} \exp \left(-\alpha r^{2}\right) \\
\hat{\psi}(\mathbf{p})=\left(\frac{1}{2 \pi \alpha}\right)^{3 / 4} \exp \left(-\frac{p^{2}}{4 \alpha}\right)
\end{gathered}
$$

the integrals in (2.2)-(2.5) can all be solved in closed form, yielding [16]

$$
\begin{gathered}
P(u)=\left(\frac{\alpha}{\pi}\right)^{3 / 2} 4 \pi u^{2} \exp \left(-\alpha u^{2}\right) \\
M(v)=\left(\frac{1}{4 \pi \alpha}\right)^{3 / 2} 4 \pi v^{2} \exp \left(-\frac{v^{2}}{4 \alpha}\right) \\
W(u, v)=\frac{2 u^{2} v^{2}}{\pi} \exp \left(-\alpha u^{2}-\frac{v^{2}}{4 \alpha}\right) \\
A(w)=\frac{2 w^{2}}{\pi} K_{0}(w)
\end{gathered}
$$

where $K_{0}$ is a modified Bessel function of the second kind.

If the orbital is an exponential function, as in the Kellner wavefunction (1.2),

$$
\begin{gathered}
\psi(\mathbf{r})=\left(\frac{\zeta^{3}}{\pi}\right)^{1 / 2} \exp (-\zeta r) \\
\hat{\psi}(\mathbf{p})=\frac{2 \sqrt{2} \zeta^{5 / 2}}{\pi\left(p^{2}+\zeta^{2}\right)^{2}}
\end{gathered}
$$




\section{GILL, BESLEY, AND O’NEILL}

the position and momentum intracules are readily obtained [21] as

$$
\begin{array}{r}
P(u)=\frac{\zeta^{3} u^{2}}{6}\left(3+6 \zeta u+4 \zeta^{2} u^{2}\right) \exp (-2 \zeta u) \\
M(v)=\frac{64 \zeta^{5}}{3 \pi} \frac{1584 \zeta^{4} v^{2}+88 \zeta^{2} v^{4}+3 v^{6}}{\left(4 \zeta^{2}+v^{2}\right)^{6}}
\end{array}
$$

but the integrals in $W(u, v)$ and $A(w)$ are more challenging. We consider the case where $\zeta=1$ because $W(u, v)$ can be obtained in the general case by the simple coordinate scaling

$$
W(u, v ; \zeta)=W\left(\zeta u, \zeta^{-1} v\right),
$$

and the action intracule is independent of $\zeta$.

We begin by writing each of the four exponentials in (2.4) as its Gaussian transform [22]

$$
\frac{\exp (-r)}{\sqrt{\pi}}=\frac{1}{\pi} \int_{0}^{\infty} \frac{1}{\alpha^{2}} \exp \left(-\frac{1}{4 \alpha^{2}}\right) \exp \left(-\alpha^{2} r^{2}\right) d \alpha,
$$

which yields

$$
\begin{aligned}
& W(u, v)=\frac{1}{\pi^{4}} \int_{0}^{\infty} \int_{0}^{\infty} \int_{0}^{\infty} \int_{0}^{\infty} \frac{1}{(\alpha \beta \gamma \delta)^{2}} \\
& \times \exp \left[-\frac{1}{4}\left(\frac{1}{\alpha^{2}}+\frac{1}{\beta^{2}}+\frac{1}{\gamma^{2}}+\frac{1}{\delta^{2}}\right)\right] W_{\alpha \beta \gamma \delta} d \alpha d \beta d \gamma d \delta,
\end{aligned}
$$

where $W_{\alpha \beta \gamma \delta}$ is the Wigner integral over Gaussians with exponents $\alpha^{2}, \beta^{2}, \gamma^{2}$, and $\delta^{2}$, which is [17]

$$
\begin{aligned}
& W_{\alpha \beta \gamma \delta}=\frac{2 \pi^{2} u^{2} v^{2}}{\left(\alpha^{2}+\delta^{2}\right)^{3 / 2}\left(\beta^{2}+\gamma^{2}\right)^{3 / 2}} \\
& \quad \times \exp \left[-\left(\frac{\alpha^{2} \delta^{2}}{\alpha^{2}+\delta^{2}}+\frac{\beta^{2} \gamma^{2}}{\beta^{2}+\gamma^{2}}\right) u^{2}\right. \\
& \left.-\left(\frac{1}{\alpha^{2}+\delta^{2}}+\frac{1}{\beta^{2}+\gamma^{2}}\right) \frac{v^{2}}{4}\right] j_{0}\left[\left(\frac{\alpha^{2}-\delta^{2}}{\alpha^{2}+\delta^{2}}-\frac{\beta^{2}-\gamma^{2}}{\beta^{2}+\gamma^{2}}\right) \frac{u v}{2}\right]
\end{aligned}
$$

If the last factor in (2.19) is written in the integral representation

$$
j_{0}(x)=\frac{1}{2} \int_{-1}^{+1} \exp (i x z) d z
$$

the integrand of (2.18) factorizes and we obtain

$$
W(u, v)=\frac{2 u^{2} v^{2}}{\pi} \int_{0}^{1} f(u, v, z)^{2} d z
$$

$$
\begin{gathered}
f(u, v, z)=\int_{0}^{\infty} \int_{0}^{\infty} \frac{\pi^{-1 / 2} x^{-2} y^{-2}}{\left(x^{2}+y^{2}\right)^{3 / 2}} \exp \left[-\frac{x^{2}+y^{2}}{4 x^{2} y^{2}}\right. \\
\left.-\frac{x^{2} y^{2} u^{2}}{x^{2}+y^{2}}-\frac{v^{2}}{4\left(x^{2}+y^{2}\right)}+\frac{x^{2}-y^{2}}{x^{2}+y^{2}} \frac{i u v z}{2}\right] d x d y .
\end{gathered}
$$

After transforming to polar coordinates, this becomes

$$
\begin{aligned}
& f(u, v, z)=\frac{2}{\sqrt{\pi}} \int_{0}^{\infty} \int_{0}^{\pi} \frac{1}{r^{6} \sin ^{2} \theta} \\
& \times \exp \left[-\frac{1}{r^{2} \sin ^{2} \theta}-\frac{u^{2} r^{2} \sin ^{2} \theta}{4}-\frac{v^{2}}{4 r^{2}}+\frac{i u v z}{2} \cos \theta\right] d \theta d r .
\end{aligned}
$$

If we integrate over $r$ and then let $x=\cos \theta$, we obtain

$$
\begin{gathered}
f(u, v, z)=\frac{3}{2} \int_{0}^{1} t^{-5}\left(1+u t+\frac{u^{2} t^{2}}{3}\right) \\
\times \exp (-u t)\left(1-x^{2}\right) \cos \left(\frac{u v x z}{2}\right) d x \\
t=\left[1+\frac{v^{2}}{4}\left(1-x^{2}\right)\right]^{1 / 2} .
\end{gathered}
$$

We have not been able to integrate (2.24) in closed form but, for specified values of $u$ and $v$, one can evaluate $W(u, v)$ to any desired accuracy by applying nested one-dimensional quadratures to (2.21) and (2.24).

The action intracule for the Kellner wavefunction is independent of $\zeta$ and is given by

$$
A(w)=\frac{2 w^{2}}{\pi} \int_{0}^{\infty} \frac{1}{u} \int_{0}^{1} f\left(u, \frac{w}{u}, z\right)^{2} d z d u .
$$




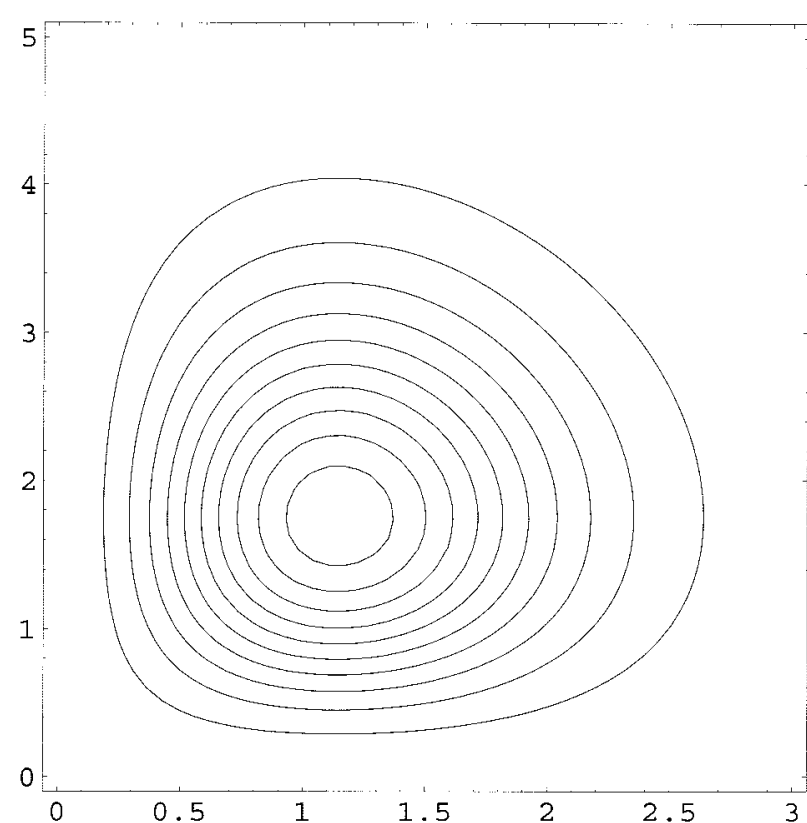

FIGURE 1. Wigner intracule $W(u, v)$ for the helium atom using a GTF orbital with $\alpha=(33-8 \sqrt{ } 2) / 9 \pi$. $u$ is plotted horizontally; $v$ is plotted vertically.

For a specified value of $w$, this can be evaluated by quadrature but, because the integrand with respect to $u$ decays slowly, accurate quadrature requires many points and is relatively slow.

\section{Results}

The Wigner intracules of atoms and molecules are positive over most of their domain, but sometimes possess small negative regions near to the origin $[16,18,19]$ that are associated with electrons of parallel spin and are therefore quasiprobability, not true probability, densities. However, although we have not obtained a closed-form expression for $W(u, v)$ in the Kellner helium-like ions, it is clear from (2.21) that, like (2.10), it is nowhere negative.

It is interesting to compare the intracules from the Gaussian orbital (2.6) with those from the exponential orbital (2.12) but it is not immediately clear which values of $\alpha$ and $\zeta$ should be employed in such a comparison. One solution is to use the values

$$
\begin{gathered}
\alpha_{\text {opt }}=\frac{33-8 \sqrt{2}}{9 \pi} \\
\zeta_{\text {opt }}=\frac{27}{16}
\end{gathered}
$$

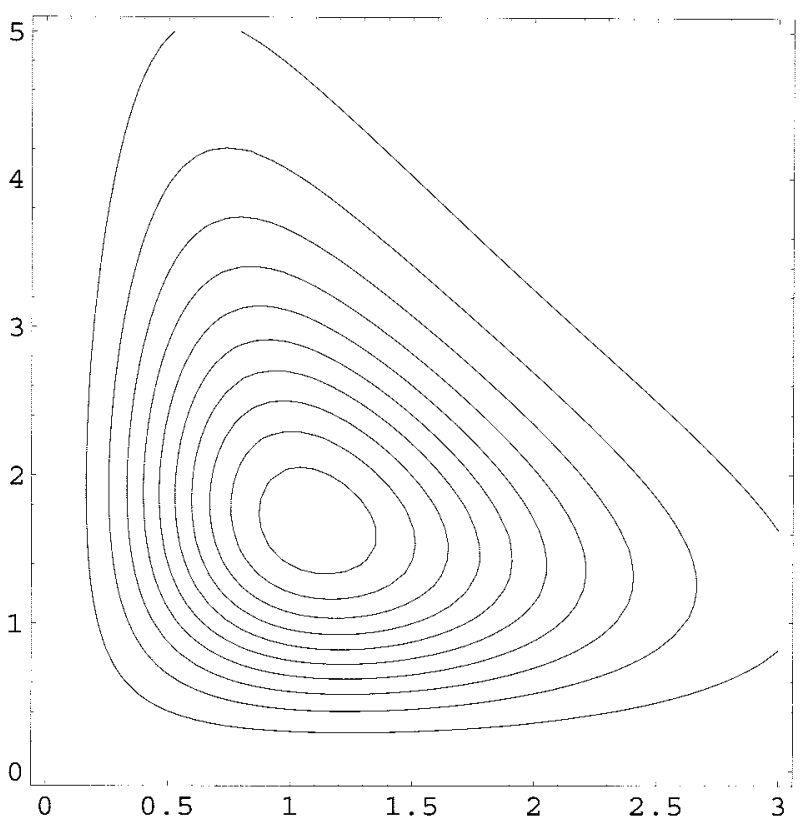

FIGURE 2. Wigner intracule $W(u, v)$ for the helium atom using a STF orbital with $\zeta=27 / 16$. $u$ is plotted horizontally; $v$ is plotted vertically.

that minimize the energy of the helium atom; Figures 1 and 2 show contour plots of the Wigner intracules (2.10) and (2.21) that result from using these exponents. Each intracule possesses only a single maximum but, although these occur at similar locations, the intracules are otherwise different. The intracule for the Kellner wavefunction decays more slowly in the $u$ direction than that for the Gaussian wavefunction, implying that the electrons are more likely to be well separated in the Kellner

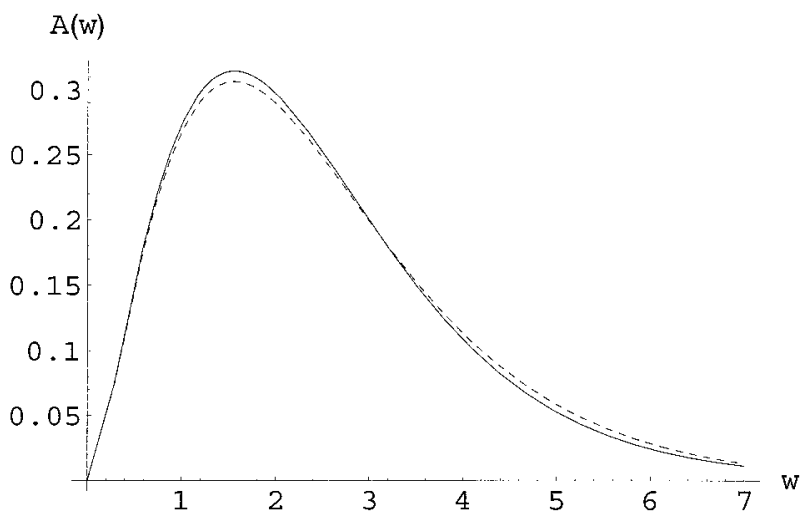

FIGURE 3. Action intracules $A(w)$ for the helium atom using: a GTF orbital with $\alpha=(33-8 \sqrt{ } 2) / 9 \pi$ (dashed line); a STF orbital with $\zeta=27 / 16$ (solid line). 
GILL, BESLEY, AND O’NEILL

TABLE I

Maxima of the Wigner and action intracules for Gaussian and Kellner wavefunctions.

\begin{tabular}{lcccrr}
\hline & \multicolumn{3}{c}{ Wigner intracule } & & \multicolumn{2}{c}{ Action intracule } \\
\cline { 2 - 5 } Orbital type & $u$ & $v$ & $W(u, v)$ & & $w$ \\
\hline GTF, Eq. (2.6) & $\alpha^{-1 / 2}$ & $2 \alpha^{1 / 2}$ & 0.3446 & 1.5527 & 0.3065 \\
STF, Eq. (2.12) & $1.8518 \zeta^{-1}$ & $0.9892 \zeta$ & 0.2824 & 1.5563 & 0.3146 \\
\hline
\end{tabular}

wavefunction and reflecting the greater diffuseness of the STF orbital. Similarly, the Kellner intracule decays more slowly in the $v$ direction than the Gaussian intracule, implying that the electrons are more likely to be found with high relative velocity in the Kellner wavefunction and reflecting the existence of cusps in the STF orbital. The shape of the Kellner intracule is, in fact, similar to that of the HF /6-311G intracule shown in Figure 3 of Ref. [16] demonstrating that the triple-zeta Gaussian basis can mimic an exponential orbital quite well.

Figure 3 shows the action intracules (2.11) and (2.26) that result from the Gaussian and Kellner wavefunctions, respectively. (As mentioned above, both intracules are independent of the exponents chosen.) The action intracule for the Kellner wavefunction reaches a slightly higher maximum than that from the Gaussian wavefunction, and then decays slightly more quickly, but their respective maxima occur at almost exactly the same value of $w$ and, in light of the significant differences between the associated Wigner intracules, the similarity between the derived action intracules is striking. The surprising insensitivity of action intracules to details of the underlying orbitals is a feature that we have noted previously [16].

The locations and magnitudes of the maxima in the Wigner and action intracules are summarized in Table I. It follows from these results that, as $\alpha$ is varied in (2.6), the maximum in the resulting Wigner intracule moves along the hyperbola $u v=2$ and, as $\zeta$ is varied in (2.12), the maximum in the resulting Wigner intracule moves along the hyperbola $u v=1.832$

\section{Conclusion}

We have shown that it is possible to compute both Wigner and action intracules for the Kellner wavefunctions of helium-like ions. Although we have not been able to write these in closed form, the integrals in Eqs. (2.21) and (2.24) submit to standard quadrature methods and provide a computationally feasible route to the evaluation of $W(u, v)$ and $A(w)$ at arbitrary points.

In order to attack the eight-dimensional integral (2.4), we have found it useful to write the STF as the Gaussian transform (2.17) and this technique [22] should be applicable to more complicated STFbased wavefunctions than those considered here. In particular, the extension to the Eckart wavefunction [8]

$$
\begin{aligned}
& \Psi\left(\mathbf{r}_{1}, \mathbf{r}_{2}\right)=\exp \left(-\alpha r_{1}\right) \exp \left(-\beta r_{2}\right) \\
&+\exp \left(-\beta r_{1}\right) \exp \left(-\alpha r_{2}\right),
\end{aligned}
$$

which allows for some radial correlation (but no angular correlation) would be straightforward using the same approach.

\section{ACKNOWLEDGMENTS}

This article was written as PMWG was enjoying an Erskine Fellowship; he expresses his thanks to the University of Canterbury for this opportunity. The work was partly supported by EPSRC grants (GR/R62052 to PMWG, GR/R77636 to NAB and GR/R81121 to DPO).

\section{References}

1. Bouferguene, A.; Weatherford, C. A.; Jones, H. W. Phys Rev E 1999, 59, 2412-2423.

2. Bouferguene, A.; Jones, H. W. J Chem Phys 1998, 109, 57185729.

3. Bouferguene, A.; Etemadi, B.; Jones, H. W. Int J Quantum Chem 1998, 70, 89-93.

4. Jones, H. W. Int J Quantum Chem 1997, 61, 881-889.

5. Kato, T. Comm Pure Appl Math 1957, 10, 151-177.

6. Agmon, S. Lectures on Exponential Decay of Solutions of Second-Order Elliptic Equations: Bounds on Eigenfunctions 
WIGNER INTRACULE FOR KELLNER HELIUM-LIKE IONS

of $N$-body Schrödinger Operators; Princeton University Press: Princeton, 1982.

7. Kellner, G. W. Z Phys 1927, 44, 91.

8. Eckart, C. Phys Rev 1930, 36, 878-892.

9. Hylleraas, E. A. Z Phys 1930, 65, 209.

10. Kinoshita, T. Phys Rev 1957, 105, 1490-1502.

11. Pekeris, C. L. Phys Rev 1958, 112, 1649-1658.

12. Thakkar, A. J.; Koga, T. Theor Chem Acc 2003, 109, 36-39 and references therein.

13. Lee, A. M.; Gill, P. M. W. Chem Phys Lett 1999, 313, 271-278.

14. Gill, P. M. W.; Lee, A. M.; Nair, N.; Adamson, R. D. J Mol Struct (Theochem) 2000, 506, 303-312.

15. Besley, N. A.; Lee, A. M.; Gill, P. M. W. Mol Phys 2002, 100, 1763-1770.
16. Gill, P. M. W.; O'Neill, D. P.; Besley, N. A. Theor Chem Acc 2003, 109, 241-250.

17. Besley, N. A.; O'Neill, D. P.; Gill, P. M. W. J Chem Phys 2003, 118, 2033-2038.

18. O'Neill, D. P.; Gill, P. M. W. Phys Rev A 2003, 68, 022505022511.

19. Besley, N. A.; Gill, P. M. W. J Chem Phys 2004, 120, 72907297.

20. O'Neill, D. P.; Gill, P. M. W. In Recent Advances in Electron Correlation Methodology; Wilson, A. K., Ed.; American Chemical Society Press: New York, 2004.

21. Koga, T.; Matsuyama, H. J Chem Phys 1997, 107, 8510-8517.

22. Boys, S. F.; Shavitt, I. University of Wisconsin Report, WISAF-13, 1959. 